\title{
ON NEUTRAL-DELAY TWO-SPECIES LOTKA-VOLTERRA COMPETITIVE SYSTEMS
}

\author{
Y. KUANG ${ }^{1}$
}

(Received 25 September 1989)

\begin{abstract}
The qualitative behavior of positive solutions of the neutral-delay two-species LotkaVolterra competitive system with several discrete delays is investigated. Sufficient conditions are obtained for the local asymptotic stability of the positive steady state. In fact, some of these sufficient conditions are also necessary except at those critical values. Results on the oscillatory and non-oscillatory characteristics of the positive solutions are also included.
\end{abstract}

\section{Introduction}

The autonomous logistic delay-differential equation

$$
\dot{x}(t)=r x(t)[1-x(t-\tau) / K],
$$

where $" "=d / d t, r, K, \tau$ are positive constants, has been widely used as a model equation capable of showing oscillations of single-species population sizes in constant environments closed to both immigration and emigration. See Cushing [3], Gopalsamy and Zhang [9], Hale [12], Kuang and Feldstein [14] and Pielou [15]. It has been the object of intensive analysis by numerous authors (see the references cited in Gopalsamy and Zhang [9]). Indeed, it is a natural generalisation of the following well-known logistic single-species population equation

$$
\dot{x}(t)=r x(t)[1-x(t) / K] .
$$

Here $r$ is called the intrinsic growth rate of the species $x, K$ is interpreted as the environment capacity for $x$, and $r[1-x(t) / K]$ is the per capita growth rate of $x$ at time $t$. Based on his investigation on laboratory populations of

'Department of Mathematics, Arizona State University, Tempe, AZ 85287-1804 U.S.A.

(C) Copyright Australian Mathematical Society 1991, Serial-fee code 0334-2700/91 
Daphnia magna, F. E. Smith [16] argued that the per capita growth rate in (1.2) should be replaced by $r[1-(x(t)+\rho \dot{x}(t)) / K]$ (for details see Pielou [15]). This leads to the following equation

$$
\dot{x}(t)=r x(t)[1-(x(t)+\rho \dot{x}(t)) / K] .
$$

We may think of $x$ as a species grazing upon vegetation, which takes time $\tau$ to recover. In this case, it will be even more realistic to incorporate a single discrete delay $\tau$ in the per capita growth rate, which results in the following neutral-delay logistic equation

$$
\dot{x}(t)=r x(t)[1-(x(t-\tau)+\rho \dot{x}(t-\tau)) / K] .
$$

This equation was introduced and investigated by Gopalsamy and Zhang [9]. Subsequently, it was studied by Freedman and Kuang [5], Kuang and Feldstein [14]. The focus of these works is the qualitative behavior of the solutions, such as boundedness, asymptotic stability and oscillation.

Assume $x(t)$ described by (1.4) is the population of a species competing with another species with population $y(t)$ for a shared limited resourcespace or a nutrient, for example, then the following system may model their interaction,

$$
\left\{\begin{array}{l}
\dot{x}(t)=r_{1} x(t)\left[1-k_{1} x(t)-\alpha x\left(t-\tau_{1}\right)-\beta \dot{x}\left(t-\tau_{0}\right)-c_{1} y\left(t-\tau_{2}\right)\right], \\
\dot{y}(t)=r_{2} y(t)\left[1-c_{2} x\left(t-\tau_{3}\right)-k_{2} y\left(t-\tau_{4}\right)\right] .
\end{array}\right.
$$

Here all parameters except $\beta$ are assumed to be positive constants. We have included $k_{1} x(t)$ into the per capita growth rate of $x(t)$, which may reflect the possible instantaneous interference within species $x$.

The focus of our present study is the qualitative behavior of positive solutions of system (1.5). This includes the asymptotic stability of its positive steady state, and oscillatory behavior of its solution about it.

This paper is organised as follows: Section 2 presents some preliminary analyses of the system. Section 3 is devoted to the asymptotic stability analysis of the unique positive steady state. The technique utilised here is a generalised version of the one developed by Cooke and Grossman [1] mainly for equations of linear constant coefficients with single delay. It is further developed by Cooke and van den Driessche [2] for several other situations. Section 4 contains a detailed oscillation analysis for both the single species equation (1.4) and the competitive (1.5). The whole paper concludes with a section of discussion.

\section{Preliminaries}

Throughout the rest of this paper, we always assume that the initial conditions for system (1.5) are of the type 


$$
\begin{array}{ll}
x(s)=\phi_{1}(s) \geq 0, & s \in[-\tau, 0], \phi_{1}(0)>0 \text { and } \phi_{1} \in C^{1}\left([-\tau, 0], R^{+}\right), \\
y(s)=\phi_{2}(s) \geq 0, & s \in[-\tau, 0], \phi_{2}(0)>0 \text { and } \phi_{2} \in C^{1}\left([-\tau, 0], R^{+}\right),
\end{array}
$$

where $\tau=\max \left\{\tau_{i} ; i=0,1, \ldots, 4\right\}$. We say $(x(t), y(t))$ is a solution of $(1.5)$ on $[-\tau, \infty)$, if both $x(t)$ and $y(t)$ are positive continuously differentiable functions and satisfy both the above initial conditions and system (1.5). It is not difficult to see that solutions of (1.5) corresponding to the initial conditions of the above type exist and are unique, and they are always positive and defined on $[0, \infty)$.

If $k_{1}+\alpha>c_{2}$ and $k_{2}>c_{1}$, then (1.5) has four steady states. They are $(0,0),\left(0, k_{2}^{-1}\right),\left(\left(k_{1}+\alpha\right)^{-1}, 0\right)$ and $\left(x^{*}, y^{*}\right), x^{*}>0, y^{*}>0$, satisfy

$$
\left\{\begin{array}{l}
\left(k_{1}+\alpha\right) x^{*}+c_{1} y^{*}=1, \\
c_{2} x^{*}+k_{2} y^{*}=1 .
\end{array}\right.
$$

In case $\tau=\beta=0$, the system (1.5) reduces to the well-studied LotkaVolterra competition model without delay (see Waltman [17]). In this situation, $\left(x^{*}, y^{*}\right)$ attracts all positive solutions of system $(1.5),(0,0)$ is an unstable node and the other two steady states are saddles. If $\tau=\beta=0$, $k_{1}+\alpha<c_{2}$ and $k_{2}<c_{1}$, then $\left(x^{*}, y^{*}\right)$ becomes unstable and both $\left(0, k_{2}^{-1}\right)$ and $\left(\left(k_{1}+\alpha\right)^{-1}, 0\right)$ become stable. In case (i) $k_{1}+\alpha>c_{2}$ and $k_{2}<c_{1}$ or (ii) $k_{1}+\alpha<c_{2}$ and $k_{1}>c_{1},\left(x^{*}, y^{*}\right)$ does not exist.

It is easy to see that the positive cone $R_{+}^{2}=\{(x, y) \mid x \geq 0, y \geq 0\}$ is invariant in the sense that if $\left(\phi_{1}(s), \phi_{2}(s)\right), s \geq 0$ is in $R_{+}^{2}$, and if $(x(t), y(t))$ is a solution of (1.5), and $x(s)=\phi_{1}(s), y(s)=\phi_{2}(s)$, then $(x(t), y(t)) \in R_{+}^{2}$ for all $t \geq s$. In a similar sense, we see that both the $x$-axis and $y$-axis are invariant as well.

Denote

$$
\|\phi\|=\max \{|\phi(s)|: s \in[-\tau, 0]\}+\max \left\{\left|\phi^{\prime}(s)\right|: s \in[-\tau, 0]\right\},
$$

then $\|\phi\|$ defines a norm for functions in $C^{1}\left([-\tau, 0], R^{+}\right)$. It is easy to see that if both $\left\|\phi_{1}\right\|$ and $\left\|\phi_{2}\right\|$ are very small, we will witness an increase in the populations of both species. This indicates that the origin has a similar property to an unstable node in the conventional sense. Suppose $\left(x_{0}, y_{0}\right)$ is a steady state of (1.5); we say it is stable if for any $\varepsilon>0$, there is a $\delta_{0}=\delta_{0}(\varepsilon)$ such that if

$$
\max \left\{\left|\phi_{1}(s)-x_{0}\right|: s \in[-\tau, 0]\right\}+\max \left\{\left|\phi_{2}(s)-y_{0}\right|: s \in[-\tau, 0]\right\}<\delta_{0},
$$

then

$$
\max \left\{\left|x(t)-x_{0}\right|: t \in R^{+}\right\}+\max \left\{\left|y(t)-y_{0}\right|: t \in R^{+}\right\}<\varepsilon .
$$


We say $\left(x_{0}, y_{0}\right)$ is unstable if $\left(x_{0}, y_{0}\right)$ is not stable. In this sense, we see that $(0,0)$ is always unstable.

It is easy to see that the properties associated with the unique positive equilibrium $\left(x^{*}, y^{*}\right)$ (if it exists) plays a central role in determining the qualitative behavior of solutions of (1.5). Consequently, it becomes the focus of our analysis in the subsequent sections.

\section{Local stability of the positive equilibrium}

In this section, we consider the local stability of the unique positive equilibrium $\left(x^{*}, y^{*}\right)$ of the neutral-delay two-species Lotka-Volterra competition system:

$$
\left\{\begin{array}{l}
\dot{x}(t)=r_{1} x(t)\left[1-k_{1} x(t)-\alpha x(t-\tau)-\beta \dot{x}(t-\tau)-c_{1} y(t)\right], \\
\dot{y}(t)=r_{2} y(t)\left[1-k_{2} y(t)-c_{2} x(t-\sigma)\right] .
\end{array}\right.
$$

The first equation of (3.1) is equivalent to

$$
\frac{d}{d t}\left[\ln \left(x(t) / x^{*}\right)+r_{1} \beta x(t-\tau)\right]=r_{1}\left[1-k_{1} x(t)-\alpha x(t-\tau)-c_{1} y(t)\right] .
$$

Denote

$$
u(t)=\ln \left(x(t) / x^{*}\right), \quad v(t)=y(t)-y^{*},
$$

then $x(t)=x^{*} \exp (u(t)), y(t)=v(t)+y^{*}$. System (3.1) is reduced to

$$
\left\{\begin{array}{l}
\frac{d}{d t}\left[u(t)+r_{1} \beta x^{*} u(t-\tau)+G_{1}(u(t-\tau))\right]=-r_{1} k_{1} x^{*} u(t)-r_{1} \alpha x^{*} u(t-\tau) \\
-r_{1} c_{1} v(t)+F_{1}(u(t), u(t-\tau), v(t)), \\
\frac{d}{d t} v(t)=-r_{2} k_{2} v(t)-r_{2} c_{2} y^{*} x^{*} u(t-\sigma)+F_{2}(u(t-\sigma), v(t)),
\end{array}\right.
$$

where $G_{1}, F_{1}, F_{2}$ satisfy

$$
\begin{aligned}
& G_{1}(0)=G_{1 u(t-\tau)}(0)=0 \\
& F_{1}(0,0,0)=F_{1 u(t)}(0,0,0)=F_{1 u(t-\tau)}(0,0,0)=F_{1 v(t)}(0,0,0)=0 \\
& F_{2}(0,0)=F_{2 u(t-\sigma)}(0,0)=F_{2 u(t)}(0,0)=0 .
\end{aligned}
$$

In the notation of Hale [12], (3.3) can be written as

$$
\frac{d}{d t}\left[D X_{t}+G\left(X_{t}\right)\right]=L X_{t}+F\left(X_{t}\right)
$$

where $X(t)=(u(t), v(t)), X_{t}=X(t+\theta), \theta \in[-\delta, 0], \delta=\max \{\tau, \sigma\}$, $X_{t} \in C\left([-\delta, 0], R^{2}\right)$, and $D, G, L, F$ are maps of $C\left([-\delta, 0], R^{2}\right)$ into 
$R^{2}$ defined by

$$
\left\{\begin{array}{l}
D X=\left(\begin{array}{c}
u(0)+r_{1} \beta x^{*} u(-\tau) \\
v(0)
\end{array}\right) \\
L X=\left(\begin{array}{c}
-r_{1} k_{1} x^{*} u(0)-r_{1} \alpha x^{*} u(-\tau)-r_{1} c_{1} v(0) \\
-r_{2} c_{2} x^{*} y^{*} u(-\sigma)-r_{2} k_{2} v(0)
\end{array}\right) \\
G(X)=\left(\begin{array}{c}
G_{1}(u(-\tau)) \\
0
\end{array}\right) \\
F X=\left(\begin{array}{c}
F_{1}(u(0), u(-\tau), v(0)) \\
F_{2}(u(-\sigma), v(0))
\end{array}\right)
\end{array}\right.
$$

It is well known that if the trivial solution of the linear system

$$
\frac{d}{d t} D X_{t}=L X_{t}
$$

is uniformly asymptotically stable, then, with $G(X)$ and $F(X)$ defined as in (3.5), the trivial solution of the nonlinear system (3.3) is (locally) exponentially asymptotically stable (for details, see Hale, [12], Theorem 9.1, pp. 304-305). Therefore, in the rest of this section, we shall consider the stability of the trivial solution of (3.6). Denote

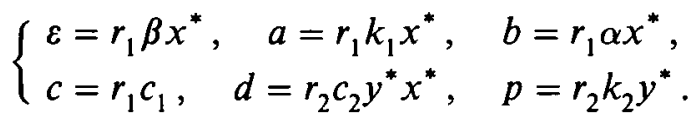

System (3.6) becomes

$$
\left\{\begin{array}{l}
\dot{u}(t)+\varepsilon \dot{u}(t-\tau)=-a u(t)-b u(t-\tau)-c v(t), \\
\dot{v}(t)=-d u(t-\sigma)-p v(t)
\end{array}\right.
$$

Differentiating both sides of the first equation of (3.8), and using the second equation leads to

$\ddot{u}(t)+\varepsilon \ddot{u}(t-\tau)+(a+p) \dot{u}(t)+(b+p \varepsilon) \dot{u}(t-\tau)+p a u(t)+p b u(t-\tau)-c d u(t-\sigma)=0$.

The second equation of (3.2) implies

$$
u(t)=-d^{-1}(\dot{v}(t+\sigma)+p v(t+\sigma)) .
$$

By substituting (3.10) into the first equation of (3.8), we obtain $\ddot{v}(t)+\varepsilon \ddot{v}(t-\tau)+(a+p) \dot{v}(t)+(b+p \varepsilon) \dot{v}(t-\tau)+p a v(t)+p b v(t-\tau)-c d v(t-\sigma)=0$. Therefore, $u(t)$ and $v(t)$ have the same local stability.

It is well known that the stability of the trivial solutions of an autonomous differential difference equation depends on the location of the roots of its characteristic equation. If the characteristic equation associated with a linear neutral equation has roots only with negative real parts, and if all the 
roots are uniformly bounded away from the imaginary axis, then the trivial solution of the linear neutral equation is uniformly asymptotically stable in the sense of Hale [12] (for details, see Hale, [12], Corollary 10.1, p. 310). The characteristic equation of (3.9) is

$$
\lambda^{2}+\varepsilon \lambda^{2} e^{-\lambda \tau}+(a+p) \lambda+(b+p \varepsilon) \lambda e^{-\lambda \tau}+p a+p b e^{-\lambda \tau}-c d e^{-\lambda \sigma}=0 .
$$

Before we state and prove our results, we need the following theorem which is essentially the same as Theorem 4.1 in Freedman and Kuang [5]. In the rest of this section, stability is referred to as the uniform asymptotic stability, unless stated otherwise. Therefore, the stability of the trivial solution of (3.8) implies the local stability of the trivial solution of (3.3), which is equivalent to the local stability of the positive equilibrium of the original system (3.1).

Theorem 3.1 (Freedman and Kuang). Consider the following second-order real scalar linear neutral-delay equation

$$
\ddot{u}(t)+\varepsilon \ddot{u}(t-\tau)+\bar{a} \dot{u}(t)+\bar{b} \dot{u}(t-\tau)+\eta u(t)+\xi u(t-\tau)=0 .
$$

Assume $|\varepsilon|<1, \eta+\xi \neq 0$ and $\bar{a}^{2}+\bar{b}^{2}+(\xi-\varepsilon \eta)^{2} \neq 0$. Then the number of different positive (negative) imaginary roots of the characteristic equation of (3.12)

$$
\lambda^{2}+\varepsilon \lambda^{2} e^{-\lambda \tau}+\bar{a} \lambda+\bar{b} \lambda e^{-\lambda \tau}+\eta+\xi x^{-\lambda \tau}=0
$$

can only be zero, one or two. For (3.12), the following statements are true

(1) If there are no such roots, then the stability of the trivial solution does not change for any $\tau \geq 0$.

(2) If there is one imaginary root, then an unstable trivial solution at $\tau=$ 0 never becomes stable for any $\tau \geq 0$. If the trivial solution is uniformly asymptotically stable for $\tau=0$, then it is uniformly asymptotically stable up to the delay time $\tau_{0,1}$, and becomes unstable afterwards.

(3) If there are two imaginary roots, $i \omega_{+}$and $i \omega_{-}$, such that $\omega_{+}>\omega_{-}>$ 0 , then the stability of the zero trivial solution can change a finite number of times at most as $\tau$ is increased, and eventually it becomes unstable.

(4)

$$
\operatorname{sign}\left\{\frac{d(\operatorname{Re} \lambda)}{d \tau}\right\}_{\lambda=i \omega}=\operatorname{sign}\left\{\bar{a}^{2}+2 \varepsilon \xi-2 \eta-\bar{b}^{2}+2 \omega^{2}\left(1-\varepsilon^{2}\right)\right\} \text {. }
$$

In the above statements, $\omega_{+}, \omega_{-}, \tau_{0,1}$ are defined as (if they do exist):

$$
\begin{gathered}
\omega_{ \pm}^{2}=\frac{1}{2}\left(1-\varepsilon^{2}\right)^{-1}\left\{\left(\bar{b}^{2}+2 \eta-\bar{a}^{2}-2 \xi \varepsilon\right) \pm\left[\left(\bar{b}^{2}+2 \eta-\bar{a}^{2}-2 \xi \varepsilon\right)^{2}\right.\right. \\
\left.\left.-4\left(1-\varepsilon^{2}\right)\left(\eta^{2}-\xi^{2}\right)\right]^{1 / 2}\right\} \\
\tau_{0,1}=\theta_{1} / \omega_{+},
\end{gathered}
$$


where $0 \leq \theta_{1}<2 \pi$, and

$$
\begin{aligned}
& \cos \theta_{1}=-\frac{\bar{a} \bar{b} \omega_{+}^{2}+\left(\eta-\omega_{+}^{2}\right)\left(\xi-\varepsilon \omega_{+}^{2}\right)}{\bar{b}^{2} \omega_{+}^{2}+\left(\xi-\varepsilon \omega_{+}^{2}\right)^{2}}, \\
& \sin \theta_{1}=\frac{\left(\xi-\varepsilon \omega_{+}^{2}\right) \bar{a} \omega_{+}-\bar{b} \omega_{+}\left(\eta-\omega_{+}^{2}\right)}{\bar{b}^{2} \omega_{+}^{2}+\left(\xi-\varepsilon \omega_{+}^{2}\right)^{2}} .
\end{aligned}
$$

Now we are ready to formulate our results. We shall discuss only two cases, namely (i) $\sigma=0$; (ii) $\sigma=\tau$. Our first theorem deals with the case (i) $\sigma=0$. We denote $\Delta_{1}=(b+p \varepsilon)^{2}+2(p a-c d)-(a+p)^{2}-2 p b \varepsilon$, $\Delta_{2}=4\left(1-\varepsilon^{2}\right)\left[(p a-c d)^{2}-p^{2} b^{2}\right]$.

THEOREM 3.2. In (3.8), assume $\sigma=0,|\varepsilon|<1$. Then the following statements are true.

(1) Suppose either $\Delta_{1} \leq 0$, or $\Delta_{1}^{2}<\Delta_{2}$.

(1A) If $k_{1}+\alpha>c_{2}$ and $k_{2}>c_{1}$, then the trivial solution of (3.8) is uniformly asymptotically stable for all $\tau \geq 0$.

(1B) If $k_{1}+\alpha<c_{2}$ and $k_{2}<c_{1}$, then the trivial solution of (3.8) is unstable for all $\tau \geq 0$.

(2) Suppose $\Delta_{1}>0$, and $\Delta_{1}^{2}>\Delta_{2}$.

(2A) If $k_{1}+\alpha>c_{2}$ and $k_{2}>c_{1}$, then the stability of the trivial solution of (3.8) will change from stability to instability a finite number of times as $\tau$ is increased and eventually it becomes unstable.

(2B) If $k_{1}+\alpha<c_{2}$ and $k_{2}<c_{1}$, then the stability of the trivial solution of (3.8) can change from instability to stability a finite number of times as $\tau$ is increased and eventually it becomes unstable.

Proof. When $\sigma=0$, the characteristic equation of (3.8) becomes

$$
\lambda^{2}+\varepsilon \lambda^{2} e^{-\lambda \tau}+(a+p) \lambda+(b+p \varepsilon) \lambda e^{-\lambda \tau}+(p a-c d)+p b e^{-\lambda \tau}=0 .
$$

Comparing to (3.13), we have $\bar{a}=a+p, \bar{b}=b+p \varepsilon, \eta=p a-c d, \xi=p b$.

When $\tau=\sigma=0$ in (3.8), it reduces to

$$
\left\{\begin{array}{l}
(1+\varepsilon) \dot{u}(t)=-(a+b) u(t)-c v(t), \\
\dot{v}(t)=-d u(t)-p v(t)
\end{array}\right.
$$

It is easy to see that the positive steady state $\left(x^{*}, y^{*}\right)$ of (3.1) exists if and only if $\left(1^{\circ}\right) k_{1}+\alpha>c_{2}$ and $k_{2}>c_{1}$ or $\left(2^{\circ}\right) \quad 0<k_{1}+\alpha<c_{2}$ and $k_{2}<c_{1}$. In case of $\left(1^{\circ}\right)$, the trivial solution of $(3.20)$ is asymptotically stable, and in case of $\left(2^{\circ}\right)$, it is unstable. 
Obviously, in both case $\left(1^{\circ}\right)$ and $\left(2^{\circ}\right)$, we have $\eta+\xi \neq 0 . \bar{a}^{2}+\bar{b}^{2}+$ $(\xi-\varepsilon \eta)^{2} \neq 0$. Suppose either $\Delta_{1} \leq 0$ or $\Delta_{1}^{2}<\Delta_{2}$, then (3.15) indicates that $\omega_{ \pm}$do not exist. Therefore, the first part of Theorem 3.1 can be applied to this situation, which results in the conclusion of parts (1A) and (1B).

Suppose $\Delta_{1}>0$ and $\Delta_{1}^{2}>\Delta_{2}$. Then, from (3.15), we see that both $\omega_{+}$ and $\omega_{-}$exist. Hence, parts (2) and (3) of Theorem 3.1 apply. A similar argument as made in the proof of part (1A) and (1B) can be repeated, thus proving the statement of part (2).

In (3.12), we denote

$$
\tau_{n, 1}=\frac{\theta_{1}}{\omega_{+}}+\frac{2 n \pi}{\omega_{+}}
$$

where $\theta_{1}$ is defined by (3.17) and (3.18). We also denote

$$
\tau_{n, 2}=\frac{\theta_{2}}{\omega_{-}}+\frac{2 n \pi}{\omega_{-}}
$$

where $0 \leq \theta_{2}<2 \pi$, and

$$
\begin{aligned}
& \cos \theta_{2}=-\frac{\bar{a} \bar{b} \omega_{-}^{2}+\left(\eta-\omega_{-}^{2}\right)\left(\xi-\varepsilon \omega_{-}^{2}\right)}{\bar{b}^{2} \omega_{-}^{2}+\left(\xi-\varepsilon \omega_{-}^{2}\right)^{2}}, \\
& \sin \theta_{2}=\frac{\left(\xi-\varepsilon \omega_{-}^{2}\right) \bar{a} \omega_{-}-\bar{b} \omega_{-}\left(\eta-\omega_{-}^{2}\right)}{\bar{b}^{2} \omega_{-}^{2}+\left(\xi-\varepsilon \omega_{-}^{2}\right)^{2}} .
\end{aligned}
$$

When the trivial solution of (3.12) is stable for $\tau=0, k$ switches from stability to instability to stability occur when the parameters are such that

$$
\tau_{0,1}<\tau_{0,2}<\tau_{1,1}<\cdots<\tau_{k-1,1}<\tau_{k-1,2}<\tau_{k, 1}<\tau_{k+1,1}<\tau_{k, 2} \cdots,
$$

or $k$ switches from instability to stability to instability may occur when

$$
\tau_{0,2}<\tau_{0,1}<\tau_{1,2}<\cdots<\tau_{k-1,2}<\tau_{k-1,1}<\tau_{k, 1}<\tau_{k, 2} \cdots,
$$

when the trivial solution of (3.12) is unstable when $\tau=0$ (for details, see Freedman and Kuang, [5]).

A similar result to Theorem 3.2 can be established for the case (ii) $\sigma=\tau$. In order to simplify the presentation, we denote

$$
\begin{aligned}
& \delta_{1}=(b+p \varepsilon)^{2}+2 p a-(a+p)^{2}-2 \varepsilon(p b-c d), \\
& \delta_{2}=4\left(1-\varepsilon^{2}\right)\left[p^{2} a^{2}-(p b-c d)^{2}\right] .
\end{aligned}
$$

THEOREM 3.3. In (3.8), assume $\sigma=\tau,|\varepsilon|<1$.

(1) If $\delta_{1} \leq 0$ or $\delta_{1}^{2}<\delta_{2}$, then we have the same conclusions as (1A) and (1B) in Theorem 3.2. 
(2) If $\delta_{1}>0$ and $\delta_{1}^{2}>\delta_{2}$, then we have the same conclusions as (2A) and (2B) in Theorem 3.2.

Proof. When $\sigma=\tau$, the characteristic equation of (3.8) becomes

$$
\lambda^{2}+\varepsilon \lambda^{2} e^{-\lambda \tau}+(a+p) \lambda+(b+p \varepsilon) \lambda e^{-\lambda \tau}+p a+(p b-c d) e^{-\lambda \tau}=0 .
$$

The rest of the proof is similar to the proof of Theorem 3.2, we omit the details to avoid repetition.

It is well known that when $|\varepsilon|>1$ in (3.13), then for all $\tau>0$, there is an infinite number of roots of (3.13) whose real parts are positive. In other words, this asserts that the trivial solution of (3.14) is unstable (for details, see Theorem 2.1 in Freedman and Kuang [5], or Corollary 7.1 in Hale [12], p. 29). This fact leads to the following theorem.

TheOREM 3.4. In (3.8), if $|\varepsilon|>1$, then for all $\tau>0$, its trivial solution is always unstable.

One of the results obtained from the critical case analysis for the secondorder neutral-delay equation made in Freedman and Kuang [5] is equivalent to the following theorem.

Theorem 3.5. Assume $|\varepsilon|=1$ in (3.8).

(1) Suppose $\sigma=\tau, a+p \geq \sqrt{2 p a+2|p b-c d|+(b+p \varepsilon)^{2}}$ and $p a>$ $|p b-c d|$. Then the trivial solution of (3.8) is always uniformly asymptotically stable.

(2) Suppose $\sigma=0, a+p \geq \sqrt{2(p a-c d)+2 p b+(b+p \varepsilon)^{2}}$ and $p a-c d>$ $p b$. Then the trivial solution of (3.8) is always uniformly asymptotically stable.

Proof. The detail can be found in Freedman and Kuang [5].

When $\beta=0,(3.1)$ reduces to a single discrete-delay competition model. If $\alpha \neq 0, \sigma=0$ (or $\tau)$ and $\Delta_{1} \leq 0$ or $\Delta_{1}^{2}<\Delta_{2}\left(\delta_{1} \leq 0\right.$ or $\left.\delta_{1}^{2}<\delta_{2}\right)$, then no stability switciiing can occur as $\tau$ changes its value. However, if $\sigma=0$ (or $\tau$ ), $\Delta_{1}>0$ and $\Delta_{1}^{2}>\Delta_{2}\left(\delta_{1}>0\right.$ and $\left.\delta_{1}^{2}>\delta_{2}\right)$, then the length of delay $\tau$ will play an important role in the stability of the trivial solution of system (3.8). In some circumstances, increases in the delay $\tau$ can even stabilise the unstable trivial solution. However, if $\tau$ is large enough, the presence of delay will generally destabilise the trivial solution of system (3.8), thus destabilising system (3.1).

The role played by the neutral term $\beta \dot{x}(t-\tau)$ seems quite complicated as well. In case $\sigma=0$, let $\Delta_{1}(\varepsilon) \equiv(b+p \varepsilon)^{2}+2(p a-c d)-(a+p)^{2}-2 p b \varepsilon$, 
$\Delta_{2}(\varepsilon)=4\left(1-\varepsilon^{2}\right)\left[(p a-c d)^{2}-p^{2} b^{2}\right]$. We see, $\Delta_{1}(\varepsilon)$ is increasing with respect to $\varepsilon$, for $\varepsilon \geq 0$, while $\Delta_{2}(\varepsilon)$ is decreasing with respect to $|\varepsilon|$. Suppose $\Delta_{1}(0) \leq 0$ and $\Delta_{1}(1)>0$, then increase in the value $\beta$ will result in $\Delta_{1}(\varepsilon)>$ 0 and $\Delta_{1}^{2}(\varepsilon)>\Delta_{2}(\varepsilon)$ for some value $\beta$. This makes the local stability of $\left(x^{*}, y^{*}\right)$ in (3.1) become $\tau$ dependent. If $k_{1}+\alpha>c_{2}$ and $k_{2}>c_{1}$, then the neutral term will eventually play the destabilising role as $\tau$ increases. If $k_{1}+\alpha<c_{2}$ and $k_{2}<c_{1}$, then for some values of $\tau$, the neutral term can play a stabilising role to the local stability of the steady state $\left(x^{*}, y^{*}\right)$ in system (3.1). Similar behaviours of the neutral term can appear in the case $\sigma=\tau$.

\section{Oscillatory results}

This section is devoted to the discussion of oscillatory behavior of positive solutions of the following neutral-delay Lotka-Volterra competition system with several discrete delays,

$$
\begin{aligned}
& \dot{x}(t)=r_{1} x(t)\left[1-k_{1} x(t)-\alpha x\left(t-\tau_{1}\right)-\beta \dot{x}\left(t-\tau_{0}\right)-c_{1} y\left(t-\tau_{2}\right)\right], \\
& \dot{y}(t)=r_{2} y(t)\left[1-c_{2} x\left(t-\tau_{3}\right)-k_{2} y\left(t-\tau_{4}\right)\right],
\end{aligned}
$$

where $r_{1}, r_{2}, k_{1}, k_{2}, c_{1}$ and $c_{2}$ are positive constants, $\tau_{i} \geq 0, i=0,1, \ldots$, 4. It is easy to see that when $\tau_{0}=\tau_{1}=\tau, \tau_{2}=\tau_{3}=\tau_{4}=0,(4.1)$ reduces to (3.1). In fact, as will be pointed out later, the method to be employed in the proof of our oscillatory results can be applied to systems more general than (4.1).

If $\beta=0$, the results in the previous section indicate that for some parameters $r_{1}, r_{2}, k_{1}, k_{2}, c_{1}$ and $c_{2}$ when $\tau$ increases from zero, the local stability of the positive steady state may switch. By part (4) of Theorem 3.1 and the well-known Hopf bifurcation theorem (see Theorem 1.1, p. 246 in Hale [12]), we know that the periodic solution may bifurcate from the equilibrium, thus causing oscillations. Our numeric results indicate that the presence of the neutral term can cause oscillations as well, which coincides with our local stability results, established in Section three. However, we are not sure that some of these oscillatory solutions are periodic solutions, since so far no Hopf-bifurcation theory is available to nonlinear neutral systems. Nevertheless, the following oscillatory results may provide some partial remedy to this awkward situation.

Recall that a positive function $x(t)$ defined on $[-T, \infty)$ is said to be oscillatory about $x^{*}$ if there exists a sequence $\left\{t_{n}\right\} \rightarrow \infty$ as $n \rightarrow \infty$, for which $x\left(t_{n}\right)=x^{*}, n=1,2, \ldots$. 
THEOREM 4.1. In (4.1), assume $\beta>0, \alpha+k_{1}>c_{2}, k_{2}>c_{1}$ and $\left(x^{*}, y^{*}\right)$ is the unique positive steady state. If $(x(t), y(t))$ is a bounded positive solution and $y(t)$ is not oscillatory about $y^{*}$, then $\lim _{t \rightarrow \infty} y(t)=y^{*}$ and either $x(t)$ is oscillatory about $x^{*}$, or $\lim _{t \rightarrow \infty}(x(t), y(t))=\left(x^{*}, y^{*}\right)$.

Proof. It is easy to see that the second equation of (4.1) implies $y(t)=y\left(t_{0}\right) \exp \left\{-r_{2}\left[c_{2} \int_{t_{0}}^{t}\left(x\left(s-\tau_{3}\right)-x^{*}\right) d s+k_{2} \int_{t_{0}}^{t}\left(y\left(s-\tau_{4}\right)-y^{*}\right) d s\right]\right\}$.

Since $y(t)$ is not oscillatory about $y^{*}$, we have the two possibilities

(i) $y(t)>y^{*}$ eventually;

(ii) $y(t)<y^{*}$ eventually.

We consider first the case (i). Define

$$
\begin{aligned}
& X\left(t_{1}, t_{2}\right)=\int_{t_{1}}^{t_{2}}\left(x(s)-x^{*}\right) d s, \\
& Y\left(t_{1}, t_{2}\right)=\int_{t_{1}}^{t_{2}}\left(y(s)-y^{*}\right) d s .
\end{aligned}
$$

Without loss of generality, we may assume when $t \geq t_{0}, y(t)>y^{*}$. Hence for $t_{2}>t_{1}>t_{0}, y\left(t_{1}, t_{2}\right)>0$ and $y\left(t_{1}, t_{2}\right)$ is strictly increasing with respect to $t_{2}$. Thus there are two possibilities to be considered:

(ia) $\lim _{t_{2} \rightarrow \infty} y\left(t_{1}, t_{2}\right)$ exists and is finite.

(ib) $\lim _{t_{2} \rightarrow \infty} y\left(t_{1}, t_{2}\right)=+\infty$.

Suppose (ia) is true, then by the assumption that $(x(t), y(t))$ is bounded, we see that $\dot{y}(t)$ is bounded. Combining this with the fact that $y(t)>y^{*}$ and $\int_{t_{1}}^{t_{2}}\left(y(s)-y^{*}\right) d s$ is bounded above, we have

$$
\lim _{t \rightarrow \infty} y(t)=y^{*} \text {. }
$$

From (ia), we see

$$
\lim _{\substack{t_{1} \rightarrow \infty \\ t_{2} \rightarrow \infty}} Y\left(t_{1}, t_{2}\right)=0 \text {. }
$$

This fact together with (4.5) leads to

$$
\lim _{\substack{t_{0} \rightarrow \infty \\ t \rightarrow \infty}} \int_{t_{0}}^{t}\left(x\left(s-\tau_{3}\right)-x^{*}\right) d s=0 .
$$

It is easy to see

$$
\int_{t_{0}}^{t}\left(x\left(s-\tau_{3}\right)-x^{*}\right) d s=X\left(t_{0}-\tau_{3}, t-\tau_{3}\right),
$$


hence we have

$$
\lim _{\substack{t_{1} \rightarrow \infty \\ t_{2} \rightarrow \infty}} X\left(t_{1}, t_{2}\right)=0 \text {. }
$$

Obviously, (4.9) implies there is a sequence

$$
\bar{t}_{i}, \quad i=1,2, \ldots, \quad \lim _{i \rightarrow \infty} \bar{t}_{i}=0 \text { and } \lim _{i \rightarrow \infty} x\left(\bar{t}_{i}\right)=x^{*} .
$$

We note that the first equation of (4.1) implies

$$
\begin{gathered}
x(t)=x\left(t_{0}\right) \exp \left\{-r_{1}\left[k_{1} \int_{t_{0}}^{t}\left(x(s)-x^{*}\right) d s+d \int_{t_{0}}^{t}\left(x\left(s-\tau_{1}\right)-x^{*}\right) d s\right.\right. \\
\left.\left.+\beta\left(x\left(t-\tau_{0}\right)-x\left(t_{0}-\tau_{0}\right)\right)+c_{1} \int_{t_{0}}^{t}\left(y\left(s-\tau_{2}\right)-y^{*}\right) d s\right]\right\} .
\end{gathered}
$$

Suppose $x(t)$ is not oscillatory about $x^{*}$ and assume first that $x(t)>x^{*}$ eventually. We denote $\bar{x}=\lim \sup _{t \rightarrow \infty} x(t), \underline{x}=\liminf _{t \rightarrow \infty} x(t)$. By (4.9), we see $\bar{x} \geq x^{*}$ and $\underline{x}=x^{*}$. In the following, we want to prove that $\bar{x}=x^{*}$. Assume that $\bar{x}>x^{*}$, then there exists $\varepsilon$, such that $0<2 \varepsilon<\bar{x}-x^{*}$ and

$$
\left(x^{*}+\varepsilon\right) e^{r_{1}\left(k_{1}+|\alpha|+c_{1}\right) \varepsilon} \cdot e^{-r \beta\left(\bar{x}-x^{*}-2 \varepsilon\right)} \leq x^{*}-\varepsilon .
$$

It is easy to see that there exists $\bar{t}_{0}>0$, such that $x(t)>x^{*}, x\left(\bar{t}_{0}\right)<x^{*}+\varepsilon$, $x\left(\bar{t}_{0}-\tau_{0}\right)<x^{*}+\varepsilon$,

$$
\begin{gathered}
\left|\int_{\bar{t}_{0}}^{t}\left(x(s)-x^{*}\right) d s\right|<\varepsilon, \quad\left|\int_{\bar{t}_{0}}^{t}\left(x\left(s-\tau_{1}\right)-x^{*}\right) d s\right|<\varepsilon, \\
\left|\int_{t_{0}}^{t}\left(y\left(s-\tau_{2}\right)-y^{*}\right) d s\right|<\varepsilon
\end{gathered}
$$

for $t \geq \bar{t}_{0}$. Since $\limsup \sup _{t \rightarrow \infty} x(t)=\bar{x}>x^{*}$, we can find a $\bar{t}_{1}>\bar{t}_{0}$, such that $x\left(\bar{t}_{1}-\tau_{0}\right)>\bar{x}-\varepsilon$. For this choice of $\bar{t}_{0}, \bar{t}_{1}$, we have

$$
x\left(\bar{t}_{1}\right) \leq\left(x^{*}+\varepsilon\right) e^{r_{1}\left(k_{1}+|\alpha|+c_{1}\right) \varepsilon} \cdot e^{-r \beta\left(\bar{x}-x^{*}-2 \varepsilon\right)} \leq x^{*}-\varepsilon .
$$

This contradicts the fact that $x(t)>x^{*}$ for $t \geq \bar{t}_{0}$. Hence $\bar{x}=x^{*}$.

Now, suppose that $x(t)<x^{*}$ eventually. If $\underline{x}=\liminf _{t \rightarrow \infty} x(t)<x^{*}$, then there exists $\varepsilon_{0}$, such that $0<2 \varepsilon_{0}<x^{*}-\underline{x}$ and

$$
\left(x^{*}-\varepsilon_{0}\right) e^{-r_{1}\left(k_{1}+|\alpha|+c_{1}\right) \varepsilon_{0}} \cdot e^{r \beta\left(x^{*}-\underline{x}-2 \varepsilon_{0}\right)} \geq x^{*}+\varepsilon .
$$

A similar argument as above can show that $\underline{x}=x^{*}$. 
If (ib) is true. Then the assumption $y(t)>y^{*}$ eventually implies that the function $c_{2} X\left(t_{1}, t_{2}\right)+k_{2} Y\left(t_{1}, t_{2}\right)$ is bounded. Since

$$
\begin{aligned}
& \left(k_{1}+\alpha\right) X\left(t_{1}, t_{2}\right)+c_{1} Y\left(t_{1}, t_{2}\right) \\
& \quad=\frac{k_{1}+\alpha}{c_{2}}\left[c_{2} X\left(t_{1}, t_{2}\right)+k_{2} Y\left(t_{1}, t_{2}\right)\right]+\left[c_{1}-\frac{k_{2}}{c_{2}}\left(k_{1}+\alpha\right)\right] Y\left(t_{1}, t_{2}\right),
\end{aligned}
$$

and $c_{1}-c_{2}^{-1} k_{2}\left(k_{1}+\alpha\right)=c_{2}^{-1}\left(c_{1} c_{2}-k_{2}\left(k_{1}+\alpha\right)\right)<0$, we see from (4.10) that when $t \rightarrow+\infty, \ln x(t) \rightarrow+\infty$; thus indicating $x(t)$ is unbounded. This contradiction implies that (ib) cannot be true.

Now, assume (ii) $y(t)<y^{*}$ eventually. Again there are two possibilities to be considered:

(iia) $\lim _{t_{2} \rightarrow \infty} Y\left(t_{1}, t_{2}\right)$ exists and is finite.

(iib) $\lim _{t_{2} \rightarrow \infty} Y\left(t_{1}, t_{2}\right)=-\infty$.

The argument for the case (iia) is similar to the one for case (ia). So, in the following we assume (iib) is true. Denote $\underline{y}=\liminf _{t \rightarrow \infty} y(t)$. Obviously, $y \leq y^{*}$. Thus from (4.2), we know $c_{2} X\left(t_{1}, t_{2}\right)+k_{2} Y\left(t_{1}, t_{2}\right)$ must be bounded from below. A similar equation to (4.13) can be established in this case, which leads to the conclusion that $\ln x(t) \rightarrow-\infty$; i.e. $x(t) \rightarrow 0$ as $t \rightarrow$ $\infty$. This obviously contradicts the fact that $c_{2} X\left(t_{1}, t_{2}\right)+k_{2} Y\left(t_{1}, t_{2}\right)$ must be bounded from below. Therefore, (iib) cannot be true. This proves the theorem.

When $\alpha+k_{1}<c_{2}$ and $k_{2}<c_{1}$, the situation becomes very complicated. Even in the case that no delay exists and $\beta=0$, the limit sets of the positive solutions consist of three equilibrium states $\left(0, k_{2}^{-1}\right),\left(x^{*}, y^{*}\right)$ and $\left(\left(k_{1}+\right.\right.$ $\left.\alpha)^{-1}, 0\right)$. With the presence of delays, periodic solutions around each of the three equilibria can exist, thus making the behavior of each positive solution hard to predict. A similar result to Theorem 4.1 for this case will be very lengthy and the proof of which can be very tedious, although the method will be more or less the same. For these reasons, we choose to omit it here.

By virtue of the proof of Theorem 4.1, the following theorem is essentially proved.

THEOREM 4.2. Assume $\beta>0, \alpha \geq 0$, then every non-oscillatory positive solution of

$$
\dot{x}(t)=r_{1} x(t)\left[1-k_{1} x(t)-\alpha x\left(t-\tau_{1}\right)-\beta \dot{x}\left(t-\tau_{0}\right)\right],
$$

satisfies $\lim _{t \rightarrow \infty} x(t)=\left(k_{1}+\alpha\right)^{-1}$.

Proof. Assume first that $x(t)$ is a non-oscillatory positive solution of (4.14) which is eventually bigger than $\left(k_{1}+\alpha\right)^{-1}$. Therefore, there exists a $t_{1}$ such 
that when $t \geq t_{1}, x(t)>\left(k_{1}+\alpha\right)^{-1}$. We denote

$$
f\left(t_{1}, t\right)=\int_{t_{1}}^{t}\left[x(s)-\left(k_{1}+\alpha\right)^{-1}\right] d s .
$$

For $t \geq t_{1}, f\left(t_{1}, t\right)$ is positive and increasing. We claim that $x(t)$ must be bounded. Since otherwise, there exists $t^{*}>t_{1}$, such that

$$
x\left(t^{*}-\tau_{0}\right)>x\left(t_{1}-\tau_{0}\right)+(r \beta)^{-1}\left[\ln \left(x\left(t_{1}\right)\right)-\ln \left(\left(k_{1}+\alpha\right)^{-1 / 2}\right)\right] .
$$

This implies that

$$
\begin{aligned}
x\left(t^{*}\right) & =x\left(t_{1}\right) e^{r \int_{t_{1}}^{t^{*}}\left[1-k_{1} x(s)-d x\left(s-\tau_{1}\right)\right] d s} \cdot e^{-r \beta\left[x\left(t^{*}-\tau_{0}\right)-x\left(t_{1}-\tau_{0}\right)\right]} \\
& \leq x\left(t_{1}\right) e^{-\left[\ln \left(x\left(t_{1}\right)-\ln \left(\frac{1}{2}\left(k_{1}+\alpha\right)^{-1}\right)\right]\right.}=\left(k_{1}+\alpha\right)^{-1 / 2},
\end{aligned}
$$

which contradicts the assumption that $x(t)>\left(k_{1}+\alpha\right)^{-1}$ for $t \geq t_{1}$.

Obviously, the boundedness of $x(t)$ implies that $\lim _{t \rightarrow \infty} f\left(t_{1}, t\right)$ exists and is finite. Hence

$$
\liminf _{t \rightarrow \infty} x(t)=\left(k_{1}+\alpha\right)^{-1} .
$$

The rest of the proof is similar to the one for Theorem 4.1, we omit it to avoid repetition.

This theorem generalises Theorem 4.2 in [13]. A similar generalisation can be made to Theorem 4.1 in [13] as well.

It is conceivable that both the two possibilities in Theorem 4.1 can actually occur. Imagine that we start with the following uncoupled system

$$
\begin{aligned}
\dot{x}(t) & =r_{1} x(t)(1-x(t-1)), \\
\dot{y}(t) & =r_{2} y(t)(1-y(t)),
\end{aligned}
$$

where $r_{1}>0, r_{2}>0$. If $r_{1}>\pi / 2$, then it is well known that (see Hale [12]) $x(t)=1$ is surrounded by at least one periodic solution and $y(t)=1$ is globally stable with respect to the positive solutions of (4.18). When we couple the system

$$
\left\{\begin{array}{l}
\dot{x}(t)=r_{1} x(t)(1-x(t-1)-\varepsilon y(t)), \\
\dot{y}(t)=r_{2} y(t)(1-y(t)),
\end{array}\right.
$$

and let $\varepsilon$ increase from zero, we may see a trajectory $(x(t), y(t))$, such that $\lim _{t \rightarrow \infty} y(t)=y^{*}(\varepsilon)$, and $x(t)$ is oscillatory about $x^{*}(\varepsilon)$, where $\left(x^{*}(\varepsilon)\right.$, $\left.y^{*}(\varepsilon)\right)$ is the unique positive steady state of (4.19). When there are no delays, 
$\beta=0, \alpha+k_{1}>c_{2}$ and $k_{2}>c_{1}$, it is well-known that for any positive solution $(x(t), y(t))(4.1)$, we have $\lim _{t \rightarrow \infty}(x(t), y(t))=\left(x^{*}, y^{*}\right)$.

\section{Discussion}

In order to apply the recent results obtained by Freedman and Kuang [5], we have purposely chosen $\sigma=0$ or $\tau$. Although the method utilised by Freedman and Kuang can be extended to establish more general results to be applied to system (3.1), the computation required by this process is expected to be very tedious.

As far as only asymptotical stability and oscillation analysis are concerned, we do not have to choose the logistic per capita growth rate for both species $x$ and $y$ in order to obtain similar results to those established so far. We can replace it by some more general growth rate functions. In Section 4, if we replace $\alpha x\left(t-\tau_{1}\right)$ by $\sum_{i=1}^{n_{1}} \alpha_{i} x\left(t-\tau_{1 i}\right), \beta \dot{x}\left(t-\tau_{0}\right)$ by $\sum_{l=1}^{n_{0}} \beta_{i} \dot{x}\left(t-\tau_{0 i}\right)$, $c_{1} y\left(t-\tau_{2}\right)$ by $\sum_{t=1}^{n_{2}} c_{1 i} y\left(t-\tau_{2 i}\right), c_{2} x\left(t-\tau_{3}\right)$ by $\sum_{t=1}^{n_{3}} c_{2 i} x\left(t-\tau_{3 i}\right)$ and $k_{2} y\left(t-\tau_{4}\right)$ by $\sum_{i=1}^{n_{4}} k_{2 i} y\left(t-\tau_{4 i}\right)$ (as long as all these constants are nonnegative), then both Theorem 4.1 and Theorem 4.2 are still true. The proofs are similar but their presentations are more tedious.

As pointed out at the end of Section 3, the role played by the neutral term $\beta \dot{x}\left(t-\tau_{0}\right)$ in the per capita growth rate for species $x$ is very complex. It may serve as both a stabilising and a destabilising factor, depending on the specific situation. At the very least, we know its presence causes various difficulties for our analysis.

It is well-known and easy to prove that when $\beta=0$, then solutions of the system (1.5) (see Gopalsamy et al. [8]) are eventually uniformly bounded. With the neutral term introduced into the per capita growth rate, this is no longer true. As pointed out by Kuang and Feldstein [14], for some negative number $\beta, x(t)$ may indeed become unbounded.

One of the most important questions left untouched in this paper is what is the criterion for the existence of periodic solutions in (1.5). In fact, so far, we do not even know what the condition is for a Hopf bifurcation to occur in (1.5). Although our numerical results indicate that a similar Hopf bifurcation theorem may be true for the nonlinear neutral-delay system (1.5), we cannot provide any rigorous proof for that yet. It seems that the integral manifold technique developed by Hale [10], [11], [12] may contribute to the solution of this general problem. In case $\beta=0$, the Hopf bifurcation of system (1.5) was analysed by Gopalsamy and Aggarwala [7], and Cushing [3].

Another interesting question remaining to be investigated is what are the conditions for the global stability of the unique positive steady state in (1.5). 
In case $\beta=0$, some results are available, for instance, if $k_{1}>\alpha+c_{1}$, $\tau_{3}=0, k_{2}>c_{2}$, then $\left(x^{*}, y^{*}\right)$ is globally asymptotically stable. For linear neutral differential systems, a simple criterion for this was obtained by Gopalsamy [6].

Finally, we would like to admit that the way we have introduced the delays in (2.1) is not the most realistic one. The best way to do so is probably to replace those discrete delays by continuously distributed delays.

\section{Acknowledgements}

This research was supported partially by a FIGA Award, and a CLAS Summer Research Award at Arizona State University.

\section{References}

[1] K. L. Cooke and Z. Grossman, "Discrete delay, distributed delay and stability switches", J. Math. Anal. Appl. 86 (1982) 529-627.

[2] K. L. Cooke and P. van den Driessche, "On zeros of some transcendental equations", Funkcialaj Ekvacioj 29 (1986) 77-90.

[3] J. M. Cushing, Integrodifferential equations and delay models in population dynamics. Lecture Notes in Biomathematics 20, (Springer-Verlag, Berlin, 1977).

[4] H. I. Freedman, Deterministic mathematical models in population ecology, (Marcel Dekker, New York, (1980).

[5] H. I. Freedman and Y. Kuang, "Stability switches in linear scalar neutral delay equations", Funkcialaj Ekvacioj, to appear.

[6] K. Gopalsamy, “A simple stability criterion for linear neutral differential systems”, Funkcialaj Ekvacioj 28 (1985) 33-38.

[7] K. Gopalsamy and B. D. Aggarwala, "Limit cycles in two species competition with time delays", J. Austral. Math. Soc. Ser. B 22 (1980) 148-160.

[8] K. Gopalsamy, M. R. S. Kulenovic and G. Ladas, "Time lags in a "food-limited" population model", Appl. Anal. 31 (1988) 225-237.

[9] K. Gopalsamy and B. G. Zhang, "On a natural delay logistic equation", Dynamics and Stability of Systems 2 (1988) 183-195.

[10] J. K. Hale, "Critical cases for neutral functional differential equations", J. Differential Equations 10 (1971) 59-82.

[11] J. K. Hale, "Behavior near constant solutions of functional differential equations", $J$. of Differential Equations 15 (1974) 278-294.

[12] J. K. Hale, Theory of functional differential equations, (Springer, New York, 1977).

[13] Y. Kuang, “On neutral delay logistic Gause-type predator-prey systems", Dynamics and Stability of Systems, to appear.

[14] Y. Kuang and A. Feldstein, "Boundedness of solutions of a nonlinear unonautonomous neutral delay equation,"J. Math. Anal. Appl., to appear.

[15] E. C. Pielou, Mathematical ecology, (Wiley Interscience, New York, 1977).

[16] F. E. Smith, "Population dynamics in Daphnia magna", Ecology 44 (1963) 651-653.

[17] P. Waltman, $A$ second course in elementary differential equations, (Academic Press, New York, 1986). 